\title{
Origine sociale des vocations canadiennes de Nouvelle-France
}

\section{Pierre Hurtubise}

Volume 45, 1978

URI : https://id.erudit.org/iderudit/1007123ar

DOI : https://doi.org/10.7202/1007123ar

Aller au sommaire du numéro

Éditeur(s)

Les Éditions Historia Ecclesiæ Catholicæ Canadensis Inc.

ISSN

0318-6172 (imprimé)

1927-7067 (numérique)

Découvrir la revue

Citer cet article

Hurtubise, P. (1978). Origine sociale des vocations canadiennes de Nouvelle-France. Sessions d'étude - Société canadienne d'histoire de l'Église catholique, 45, 41-56. https://doi.org/10.7202/1007123ar

Tous droits réservés @ Les Éditions Historia Ecclesiæ Catholicæ Canadensis Inc., 1978
Ce document est protégé par la loi sur le droit d'auteur. L’utilisation des services d'Érudit (y compris la reproduction) est assujettie à sa politique d'utilisation que vous pouvez consulter en ligne.

https://apropos.erudit.org/fr/usagers/politique-dutilisation/ 


\section{Origine sociale des vocations canadiennes de Nouvelle-France}

Entre 1650 et 1762, 841 Canadiens et Canadiennes sont devenus prêtres, religieux ou religieuses en Nouvelle-France. De ce nombre, 630 étaient des femmes, réparties dans 7 communautés hospitalières ou enseignantes à Québec, Montréal et Trois-Rivières et 211, des hommes, dont 152 prêtres séculiers, 43 prêtres réguliers et 16 frères laïcs ${ }^{1}$. On s'est très peu intéressé jusqu'ici à ce groupe social, pourtant beaucoup plus facile à circonscrire et à situer que certains autres dans la société canadienne de l'époque, sinon pour en étudier tel ou tel segment particulier ${ }^{2}$ ou formuler sur lui, à partir de données souvent très fragmentaires, un certain nombre d'hypothèses concernant la nature, les limites et les difficultés de son recrutement ${ }^{3}$. Nous voudrions tenter de combler ce vide et, par le fait même, chercher à répondre à un certain nombre d'interrogations relatives à l'origine et à la représentativité sociale du groupe en question.

Nous connaissons encore très mal la société canadienne des $\mathrm{XVII}^{\mathrm{e}}$ et $\mathrm{XVIII}{ }^{\mathrm{e}}$ siècles et, cela, de l'avis de la plupart des spécialistes, parce que nous ne nous sommes pas encore attaqués aux sources qui permettraient d'arriver à vraiment la saisir de l'intérieur.

1 Les groupes étudiés sont, du côté des femmes : les Sœurs de l'HôtelDieu, les Sœurs de l'Hôpital Général et les Ursulines de Québec, les Sœurs de l'Hôtel-Dieu, les Sœurs de la Congrégation Notre-Dame et les Sœurs de la Charité de Montréal, enfin les Ursulines de Trois-Rivières; du côté des hommes : les prêtres séculiers, les Récollets, les Jésuites et les Frères Charon. Nous n'avons pas considéré les vocations, peu nombreuses il est vrai, ne tombant pas sous ces catégories. Nous avons négligé également les cas qui ont pu exister, mais qui devaient être relativement rares, de Canadiens ou de Canadiennes entrés dans des communautés ou corps qui n'existaient pas au pays.

2 La seule étude de quelque envergure consacrée à l'un de ces sousgroupes est, à notre connaissance, celle de Micheline Dallaire, L'HôpitalGénéral de Québec, 1692-1764, Montréal, 1971.

3 Voir, par exemple, L. DECHÊNE, Habitants et marchands de Montréal au XVII siècle, Montréal-Paris, 1974, pp. 476-479. 
Des enquêtes sont en cours ${ }^{4}$ qui nous fourniront sans doute des données fondamentales sur le sujet, mais en attendant qu'elles aboutissent, il nous a semblé que l'étude d'un groupe tel que celui des gens d'Église qui constituait, après tout, une sorte de microcosme de la société de l'époque, pourrait peut-être, en plus de nous ouvrir de nouvelles perspectives sur les milieux ecclésiastiques du temps, nous aider à mieux cerner le problème de la société canadienne dans son ensemble et, partant, aider les chercheurs qui présentement s'y intéressent à formuler des hypothèses tout à la fois utiles et pertinentes à ce sujet.

\section{I - SOURCES ET MÉTHODE}

Nos sources sont, pour la plupart, relativement bien connues. Il s'agit, dans le cas des communautés religieuses de femmes, de listes nominatives publiées dans les histoires de ces diverses communautés ${ }^{5}$, avec parfois des renseignements complémentaires fournis par les archivistes de l'une ou l'autre des familles en question ${ }^{6}$. Pour les Jésuites, nous avons eu recours à Melançon ${ }^{7}$; pour les Récollets, à Lanctôt ${ }^{8}$; pour les Frères Charon, à Fauteux et

4 Par exemple, celles du groupe de recherche de Jean Blain et Louis Lavallée sur les structures sociales dans la région de Montréal au XVIII siècle, enquêtes qui exploitent surtout les archives notariales.

5 H. Casgrain, Histoire de l'Hôtel-Dieu de Québec, Québec, 1978, pp. 582-591; Mgr de Saint-Vallier et l'Hôpital général de Québec, Québec, 1882, pp. 718-733 ; Les Ursulines de Trois-Rivières depuis leur établissement jusqu'à nos jours, t. IV, 1911, pp. 478-493 ; M. Mondoux, L'Hôtel-Dieu, premier hôpital de Montréal, Montréal, 1942, pp. 388-393 ; A. FerlaNDANGers, Mère d'Youville, Vénérable Marie du Frost de la Jemmerais..., Montréal, 1945, pp. 350-351 ; Sr HenRIETTE, Histoire de la Congrégation Notre-Dame, vol. I-IV, Montréal, 1941, passim.

6 En particulier les archivistes de l'Hôtel-Dieu, des Sœurs de la Charité et de la Congrégation Notre-Dame à Montréal. Nous tenons à les remercier de même que notre collaboratrice, sœur Lucienne Plante, C.N.D., qui a fait, à notre demande, des recherches plus détaillées dans les archives de sa communauté en vue de nous aider à répondre à certaines questions particulières que nous nous étions posées.

7 A. Melançon, Liste des missionnaires jésuites en Nouvelle-France et Louisiane (1611-1800), Montréal, 1929, 98 p.

8 G. Lanctot, "Nécrologie des Récollets », dans B.R.H., 39 (1933), pp. 457-462. 
Massicotte ${ }^{9}$; pour l'ensemble du clergé, aussi bien régulier que séculier, aux listes établies par Caron ${ }^{10}$, Tanguay ${ }^{11}$, Allaire ${ }^{12}$ et Provost ${ }^{13}$.

Mais ces données seraient restées très incomplètes et parfois incertaines, sans l'apport d'un certain nombre de sources manuscrites gracieusement mises à notre disposition par divers centres d'archives religieuses ou ecclésiastiques, dont un précieux Répertoire $d u$ clergé canadien dû à la plume d'Amédée Gosselin et qui se trouve présentement aux Archives du Séminaire de Québec ${ }^{14}$.

Nous avons également eu recours à un certain nombre d'instruments connus, pour ne pas dire classiques, tels le Dictionnaire généalogique de Tanguay ${ }^{15}$ et le plus récent Dictionnaire biographique du Canada ${ }^{16}$.

À partir de ces sources, nous avons pu établir pour chacune des vocations concernées, une fiche descriptive portant mention, entre autres choses, de l'origine géographique, de l'origine sociale, $\mathrm{du}$ type de vocation choisie, des repères chronologiques indispensables, tels naissance, profession, ordination, décès, des noms des parents et grands-parents, dans ce dernier cas, maternels aussi bien que paternels.

9 A. Fauteux, L'Hôpital Général des Sæurs de la Charité..., t. I, Montréal, 1916, passim ; E.Z. MASsicotTE, «Inventaire des documents et des imprimés concernant la communauté des Frères Charon et l'Hôpital Général de Montréal sous le Régime français », dans R.A.P.Q., 1923-24, pp. 163-201. De nombreux renseignements additionnels nous ont été fournis par le Fr. Gilles Beaudet, é.c., spécialiste de la question.

10 I. Caron, "Liste des prêtres séculiers et religieux qui ont exercé le saint ministère au Canada », dans B.R.H., 47 (1941), pp. 76-78; 160-175 ; $192-201$; 225-235; 257-268; 289-299. À compléter par "Le clergé de la Nouvelle-France », ibid., 54 (1948), pp. 82-91; 216-223;230-237.

11 C. Tanguay, Répertoire général $d u$ clergé canadien, Québec, 1868 et 1893 .

12 J.B.A. AllaIRE, Dictionnaire biographique du clergé canadienfrançais, 5 vol., Montréal, 1908-1932.

13 H. Provost, Le Séminaire de Québec. Documents et biographies, Québec, 1964, pp. 415 et suiv.

14 A. Gosselin, Répertoire du clergé canadien sous le régime français, 1665-1760, A.S.Q., mss 425. Précieuse également la liste des professes (16481769) que nous a fournie l'archiviste des Ursulines de Québec.

15 C. Tanguay, Dictionnaire généalogique des familles canadiennes..., Québec, 1871-1890, 7 vol.

16 Dictionnaire biographique du Canada, vol. I-III, Québec-Toronto, 1965-1974. 
Riches et, à plus d'un titre, surprenantes données - nous y reviendrons plus loin: encore fallait-il les situer dans le cadre d'une société en pleine gestation, fille de l'Europe, mais en même temps à la recherche d'une identité propre qui ne pouvait être à la longue que nord-américaine.

On a beaucoup écrit sur le sujet, mais ni la thèse d'un Groulx, d'un Frégault et, plus récemment, d'un Eccles selon laquelle les structures hiérarchiques auraient été moins rigides en Canada que dans la métropole, ni celle d'un Munro, d'un Chambers ou encore d'un Nish qui croyaient voir dans la Nouvelle-France un modèle attardé de société féodale ${ }^{17}$, ne paraissent aujourd'hui tout à fait convaincantes. Sans doute y avait-il des différences d'une société à l'autre et les administrateurs français étaient-ils les premiers à s'en rendre compte, même si cela n'apparaissait pas toujours dans leur vocabulaire, mais il n'en demeure pas moins que, pour l'essentiel, la colonie canadienne était une société fortement hiérarchisée, en tout cas beaucoup plus qu'on l'imagine habituellement ${ }^{18}$. Tout le problème est de savoir en quoi consistait cette structure hiérarchique et sur quel type de valeurs elle se fondait.

On a beaucoup épilogué sur la noblesse de Nouvelle-France, noblesse «besogneuse » qui, à en croire certains, ne tenait à peu près pas compte de la dérogeance, s'adonnait au commerce, allant même, dans certains cas, jusqu'à mettre la main à la charrue ${ }^{19}$. Il faudrait tout d'abord s'entendre sur le sens du mot «noble » et éviter de mettre dans cette catégorie des personnages qui n'avaient droit ni au titre ni aux honneurs attachés à ce dernier. La possession d'une seigneurie ou l'exercice de certaines fonctions militaires ne conféraient pas par le fait même la noblesse. Qu'il y ait eu des gentilshommes engagés dans le commerce des fourrures, le fait est trop patent pour qu'on songe à le nier. D'ailleurs un arrêt de 1685 permettait à la noblesse de Nouvelle-France de trafiquer en gros et en détail ${ }^{20}$. Mais il s'agissait dans bien des cas de "participations », parfois occultes, à des entreprises dont la gestion immédiate

17 Voir, à ce sujet, F. OUELlET, «Officiers de milice et structure sociale au Québec (1660-1815)» (Communication au Congrès d'histoire militaire, Ottawa, août 1978), pp. 2-3.

18 Cf. L. Dechêne, Habitants et marchands de Montréal, p. 403.

19 J. Hamelin et al., Histoire du Québec, Toulouse, 1976, pp. 163164 ; M. Trudel, Initiation à la Nouvelle-France, Montréal-Toronto, 1968, p. 152 .

¿20 L. DeChÊNE, op. cit., p. 384. 
ne relevait généralement pas d'eux. C'est que, grâce à son monopole sur les principales charges militaires, grâce à son influence politique également, la noblesse était à même d'exercer une sorte de tutelle sur les pêcheries et le commerce des fourrures. En somme, elle était, de par son rang, en mesure de percevoir, en échange de la protection qu'elle accordait à la traite, une sorte de rente sur la marchandise, tout comme elle en percevait une, beaucoup plus modeste il est vrai, sur les terres soumises au régime seigneurial ${ }^{21}$. Peut-on encore parler, après cela, de noblesse commerçante? Quant aux gentilshommes-laboureurs, ils ne semblent pas avoir été légions et, comme le souligne très justement Louise Dechêne, il faut se garder de généraliser les quelques exceptions signalées dans les documents de l'époque 22 .

S'il existe une différence entre la métropole et sa colonie, ce n'est pas tant au niveau de la structure qu'il faut la chercher qu'à celui de la mobilité qui, dans l'une et l'autre de ces sociétés, comme dans toute société d'ailleurs, permettait de passer d'une catégorie sociale à une autre. Or cette mobilité, du moins jusqu'au premier quart du XVIII ${ }^{e}$ siècle, a été très grande en Nouvelle-France. Nous voyons, en effet, des fils d'artisans et de paysans accéder sans problème au notariat et, de là, aux divers offices de judicature existant dans le pays, toụt comme nous voyons de simples coureurs de bois devenir interprètes du roi, puis administrateurs, en attendant un anoblissement qui, au XVII ${ }^{e}$ siècle du moins, ne tardait généralement pas à venir. Le cas d'un Pierre Boucher devenu successivement juge, seigneur, gouverneur, puis anobli, après avoir été au départ simple domestique, est loin d'être une exception dans la Nouvelle-France d'avant $1720^{23}$. On imagine difficilement la même chose en France à la même époque.

Il ne fait pas de doute que l'argent influait sur la mobilité sociale en Nouvelle-France et jusqu'à un certain point sur le statut qui en découlait, mais il n'est pas sûr, comme certains l'ont prétendu, que ce statut ait cessé, dans la colonie, de dépendre du titre ou de l'office, pour devenir le seul produit de l'argent. En d'autres mots, qu'on y soit passé assez rapidement d'une société d'ordres à une société de classes ${ }^{24}$.

21 F. OUellet, art. cit., pp. 1-2, 41.

22 L. DECHÊNE, op. cit., p. 384.

23 M. Trudel, op. cit., p. 158.

24 J. HaMelin, op. cit., p. 165. 
Sans doute le succès financier favorisait-il, dans bien des cas, l'ascension sociale, mais de simples rapports de parentèle ou de clientèle étaient très souvent tout aussi, sinon plus efficaces ${ }^{25}$. D'ailleurs, ce qui comptait vraiment dans tout ce processus ce n'était pas tant les moyens employés que le but visé qui, lui, n'avait rien à voir avec l'argent. Nous n'en voulons pour preuve que l'acharnement avec lequel les bourgeois de Nouvelle-France, tout au long des $\mathrm{XVII}^{\mathrm{e}}$ et $\mathrm{XVIII}$ siècles, chercheront par tous les moyens possibles: mariages, exercice de certaines charges, civiles aussi bien que militaires, achat de seigneuries, à obtenir des lettres d'anoblissement, et cela même après que le roi eût pratiquement cessé, à partir de 1720, d'accorder de telles faveurs. Comme le signale Fernand Ouellet qui a étudié de plus près une des avenues empruntées par ces marchands et professionnels de la colonie pour arriver à leurs fins : "L'objectif de la majorité des bourgeois qui réussissent est d'entrer un jour dans la noblesse $26 \%$. Pourquoi ? Parce que cette noblesse représente le plus haut échelon de la société canadienne de l'époque et, à cause du caractère fortement militaire de cette société, y fait figure de classe dirigeante, au même titre que le clergé ${ }^{27}$. Voilà l'attrait, l'irrésistible attrait que le statut d'aristocrate exerce sur les hommes d'argent et de talent de la colonie.

De toute évidence, la Nouvelle-France demeure une société d'ordres ou, tout au moins, à mi-chemin de la société d'ordres et de la société de classes.

Autant d'éléments qui posent avec acuité le problème des catégories sociales dans la colonie et qui, par conséquent, conditionnent, à plus d'un titre, notre recherche. Nous avons estimé que dans l'état actuel de nos connaissances et compte tenu de ce que nous avons dit jusqu'ici, il valait mieux, pour l'époque, se limiter à deux grandes catégories : celle des "notables », comprenant nobles, officiers, professionnels et marchands ; celle du «peuple», regroupant petits commerçants, artisans, manœuvres et paysans, ou " habitants », comme on disait à l'époque ${ }^{28}$. C'est donc par rapport

25 Voir, à ce propos, F. Ouellet, art. cit., p. 17 et suiv.

26 Ibid., p. 41.

27 lbid., pp. 40-41.

28 De préférence aux catégories plus nettes et plus classiques proposées par certains historiens, mais dont l'éventail varie tellement d'un auteur à l'autre, qu'on est en droit de penser que le problème n'a pas encore trouvé 
à ces deux très larges paliers que nous essayerons de situer nos 841 prêtres, religieux et religieuses, quitte à affiner nos catégories en cours de route si jamais les besoins de l'enquête l'exigeaient.

Les sources auxquelles nous avons eu recours ne mentionnent pas toujours les statut, métier ou profession des pères et grands-pères de nos personnages. Nous avons, au total, 300 fiches dans ce cas, soit $35,7 \%$ de l'ensemble. Mais pour quiconque connaît bien l'Ancien Régime et, sur ce point, les spécialistes ne nous contrediront certainement pas, il paraît évident que le fait de ne pas mentionner le statut ou la profession dans un document officiel et nos sources, il importe de le rappeler ici, s'appuient toutes sur des documents de ce type - équivaut à un aveu d'infériorité ou de moindre estime sociale. Nous n'avons donc pas hésiter à considérer ces 300 prêtres, religieux ou religieuses, sans origine sociale connue comme étant de souche «populaire».

\section{II — LES DONNÉES STATISTIQUES}

Ceci dit, que sommes-nous en mesure d'affirmer concernant les milieux d'origine et d'éveil de nos 841 «vocations»?

Commençons par quelques données statistiques, Notre groupe, globalement pris, provient à $46,5 \%$ de familles de "notables » et $53,5 \%$ de familles du «peuple ». Si nous isolons, d'un côté, les hommes, de l'autre, les femmes, nous obtenons un rapport de 57 à 43 et de 53 à $47 \%$ respectivement en faveur des vocations de souche «populaire». Même tendance, par conséquent, dans un cas comme dans l'autre, mais avec un accent plus prononcé du côté des vocations masculines. $\mathrm{Si}$, par contre, nous regroupons nos sujets par régions d'origine, soit les gouvernements de Québec, Montréal et Trois-Rivières, nous trouvons à peu près les mêmes rapports, soit de 57 à 43 et de 51 à $49 \%$, dans les deux premiers cas, mais un rapport inverse de 64 à $36 \%$ dans le cas de TroisRivières.

Si maintenant nous considérons chacune des composantes du groupe, nous constatons que le rapport est également inversé dans

sa solution. Voir, par exemple, à ce propos, L. DeCHÊNe, Habitants et marchands de Montréal..., p. [374] et suiv.; M. TRUDEL, Initiation à la Nouvelle-France, pp. 152-157 ; M. DallaIRE, L'Hôpital-Général de Québec, p. [51] et suiv. 
au moins deux cas, soit celui des Sœurs de l'Hôpital Général et celui des Ursulines de Québec qui comptent, les premières, $49 \%$, et les secondes, $41 \%$ seulement de membres d'extraction populaire, mais, mis à part les Jésuites qui se répartissent également des deux côtés du méridien social, tous les autres corps : prêtres séculiers, Récollets, Frères Charon, Sœurs de l'Hôtel-Dieu de Québec, Ursulines de Trois-Rivières, Hospitalières, Sœurs de l'Hôtel-Dieu de Québec, Ursulines de Trois-Rivières, Hospitalières, Sœurs de la Congrégation et Sœurs de la Charité de Montréal, font pencher la balance dans la direction opposée. Le phénomène est tout particulièrement frappant dans le cas des prêtres diocésains ( 58 vs $42 \%$ ), des Récollets ( 58 vs $42 \%$ ), des Sœurs de la Congrégation (60 vs $40 \%$ ) et des Sœurs de la Charité ( 69 vs $31 \%$ ). Ce qui confirme d'ailleurs, au moins pour les trois derniers groupes mentionnés, l'impression qu'avaient toujours eu les historiens de Nouvelle-France d'une plus grande familiarité et affinité de ces trois familles avec le «peuple ${ }^{29}$.

Tout compte fait, cependant, ce sont surtout, comme région, Québec, avec ses 447 vocations soit $52 \%$ de l'ensemble, et, comme groupes, les prêtres diocésains et les Sœurs de la Congrégation totalisant à eux seuls 321 ou $38 \%$ des vocations, qui donnent à notre tableau sa coloration bien particulière et, à plus d'un titre, il faut le reconnaître, inattendue. Car, prises dans leur ensemble, nos données contredisent la plupart des hypothèses émises jusqu'ici sur l'origine sociale des vocations canadiennes d'avant $1763^{30}$. Et cela, nous tenons à le préciser, malgré que nous ayons donné à la catégorie «notables» une extension plus grande que chez certains auteurs, un Marcel Trudel par exemple, qui, selon nous, se sont laissés un peu trop guider en ce domaine par des critères d'ordre d'abord et avant tout socio-économiques ${ }^{31}$. Mais, alors, comment expliquer cet enracinement «populaire» d'une bonne majorité de nos gens d'Église, alors que tout nous inclinait jusqu'ici et, d'ailleurs, aurait dû nous incliner à penser exactement le contraire ?

Peut-être nous sommes-nous laissé un peu trop obnubiler par le problème, réel certes, mais probablement moins déterminant que nous l'imaginions, des difficultés d'accès à une vocation sacerdotale

29 Cf. M. TrudeL, op. cit., p. 263 ; Id., L'Église canadienne sous le Régime militaire, t. II, Québec, 1957, pp. 338-339; 354.

30 Voir, en particulier, L. DeCHÊNE, op. cit., pp. 476-479.

31 Cf. L. Dechêne et M. Trudel, loc. cit. 
ou religieuse en Nouvelle-France. La première difficulté évoquée et sur laquelle on se fonde habituellement pour parler d'un recrutement «élitiste» des vocations est, bien évidemment, celle de la dot, mais il en est une autre, moins souvent évoquée, mais tout aussi lourde de conséquences, concernant les vocations masculines cette fois, et plus particulièrement les vocations presbytérales, qui est le coût et peut-être faudrait-il ajouter la longueur des études requises des candidats au sacerdoce. Jusqu'à quel point ces difficultés ont-elles vraiment joué ?

\section{III — CONDITIONS D'ACCÈS ET ORIGINE SOCIALE}

Sans doute le problème de la dot ne peut-il être écarté du revers de la main. Réunir les 2000 ou 3000 livres exigées par la plupart des communautés de femmes établies dans la colonie ${ }^{32}$ ne devait pas être chose facile pour les petits commerçants, artisans ou «habitants » de l'époque, voire même pour leurs concitoyens supposément mieux nantis. Que dire des pères de famille qui voyaient deux, trois, parfois même jusqu'à six de leurs enfants opter pour la vie religieuse ou sacerdotale ${ }^{33}$ ? Il est en tout cas significatif de constater un net fléchissement des vocations féminines entre 1722 et 1732 , période durant laquelle les dots des moniales avaient été portées par ordre du roi, de 3000 à 5000 livres et celles des Sœurs de la Congrégation, jusque-là indéterminées, fixées à 2000 livres ${ }^{34}$.

Significatif également que cette dernière communauté qui avait été, dès le départ, et qui restera jusqu'au bout la moins exigeante au chapitre de la dot, soit également celle qui connaisse, et de loin, le meilleur recrutement en Nouvelle-France ${ }^{35}$. Mais, justement,

32 M. Dallaire, "Conditions requises pour devenir religieuse au XVIII' siècle ", dans L'Hôtel-Dieu de Montréal (1642-1973), Montréal, 1973, pp. 190-191; Sr HenrietTe, op. cit., t. II, p. 64.

33 Nous avons, en effet, relevé 112 foyers ayant donné chacun 2 vocations ; 33 ayant donné 3 vocations; 12, 4 vocations; 3, 5 vocations et 2, 6 vocations.

34 Sr Henriette, op. cit., t. III, pp. 313-314; J. Hamelin, Économie et société en Nouvelle-France, Québec, 1960, p. 95 ; M. DallaIRE, L'HôpitalGénéral de Québec, p. 99.

35 Soit 169 religieuses sur un total de 630 , ce qui représente un pourcentage de près de $27 \%$. 
le cas des filles de Marguerite Bourgeois, que nous avons étudié de plus près, oblige à nous demander si les normes imposées d'en-haut relativement à la dot étaient toujours fidèlement respectées par nos communautés de femmes.

Nous savons, grâce aux travaux de Micheline Dallaire, que chez les Hospitalières de Montréal et chez les Sœurs de l'Hôpital Général de Québec, la dot était rarement payée au complet et plus rarement encore comptant et qu'il fallait souvent recourir à des bienfaiteurs pour combler la différence ${ }^{36}$. Chez les Sœurs de la Congrégation, la situation était plus fluctuante encore, et cela, malgré que la dot y fût, comme nous l'avons indiqué, plus basse qu'ailleurs. Sur 74 religieuses pour lesquelles nous avons des renseignements relativement complets, 12 seulement paient la totalité de leur dot au moment de la profession, soit 2000 livres ; 21 versent un acompte s'établissant en moyenne à 654 livres, avec promesse de paiements à venir sous diverses formes, dont parfois celle de droits de succession ; 10 s'engagent pour des dots variant de 1300 à 300 livres et 28 ne s'engagent pour rien du tout, laissant à des bienfaiteurs ou à une succession à venir le soin de déterminer quelle sera leur contribution effective à la caisse de la communauté ${ }^{37}$. À noter, également, et cela vaut pour l'ensemble des vocations féminines, que les paiements sont très souvent faits en nature, sous forme de biens-fonds, voire de biens de consommation, dans bien des cas, sur-évalués ${ }^{38}$.

Il ne faut donc pas se surprendre de voir bon nombre de filles du peuple entrer dans la Congrégation Notre-Dame aux $\mathrm{XVII}^{\mathrm{e}}$ et $\mathrm{XVIII}^{\mathrm{e}}$ siècles. Fidèles à l'esprit de leur fondatrice qui aurait préféré ne pas avoir à exiger de dot ${ }^{39}$, les Sœurs de la Congrégation savaient se montrer accommodantes, chaque fois que la situation financière d'une recrue, par ailleurs bien disposée, l'exigeait. Mutatis mutandis, il devait en être un peu de même pour les autres communautés féminines de Nouvelle-France ${ }^{40}$.

36 "Conditions requises pour devenir religieuse au XVIIIe siècle », pp. 197-199; L'Hôpital-Général de Québec, pp. 110-111.

37 D'après la série des contrats de profession (CC 5499-5556) et des livres de comptes conservés aux archives de la Congrégation Notre-Dame à Montréal.

38 M. DallatRe, L'Hôpital-Général de Québec, p. 115.

39 Sr Henriette, Histoire de la Congrégation Notre-Dame, t. I, p. 136.

40 Cf. M. DallaIRE, "Conditions requises pour devenir religieuse au XVIII' siècle », p. 197 ; L'Hôpital-Général, pp. 107-108. 
L'existence d'un clergé surtout «populaire» s'explique probablement de la même façon. Peu nombreux, sans doute, étaient les fils du peuple capables de se payer de longues et coûteuses études aux Petit et Grand Séminaires de Québec ${ }^{41}$. Mais, justement, on avait prévu pour eux tout un système de bourses et de demibourses leur permettant de surmonter cet obstacle. "On entretient toujours au Séminaire, écrit en 1699 l'intendant Champigny, 40 ou 50 enfants, dont une partie paye pension, d'aucuns demi-pension, et les autres y sont par charité ; ils sont enseignés depuis les premières instructions jusqu'à la fin de la théologie dans les écoles des jésuites, où ils sont envoyés deux fois par jour ${ }^{42}$.

C'est que $\mathrm{M}^{\mathrm{gr}}$ de Laval avait prévu, dès le départ, que le Séminaire nourrirait, logerait, entretiendrait et élèverait « jusqu’à la fin de la théologie huit pauvres enfants de bonnes mœurs ${ }^{43}$ ». Mais d'autres bourses étaient venues s'ajouter par la suite et surtout le Séminaire consentait très souvent des remises de pension partielles, voire même totales, de sorte qu'un nombre grandissant d'enfants du peuple s'était vu, à partir de la fin du XVII ${ }^{\mathrm{e}}$ siècle, ouvrir l'accès au sacerdoce.

Sans doute restait-il le problème du titre clérical, c'est-à-dire de la rente dont, aux termes du droit canonique ancien, le jeune clerc devait disposer au moment de l'ordination ${ }^{44}$. Mais, là encore, on avait réussi à contourner l'obstacle en garantissant au départ à chaque prêtre ordonné pour le service de l'Église de Québec un minimum de revenus et, si nécessaire, un supplément, à même la caisse de l'évêque, voire, dans certains cas, du Séminaire ${ }^{45}$. D'où le petit nombre de titres cléricaux -16 au total pour tout le

41 La pension au Petit Séminaire fut jusqu'à 1700 de cent livres par année; après 1700 , de cent soixante-douze livres de France. N. BaILlaRgEON, Le Séminaire de Québec de 1685 à 1760, Québec, 1977, p. 206.

42 Cité par A. Gosselin, L'Église du Canada depuis Mgr de Laval jusqu'à la Conquête, 1ère partie, Québec, 1911, p. 151.

43 N. Baillargeon, op. cit., pp. 206-207. Sous Mgr de Pontbriand, 36 bourses variant de 100 à 300 francs étaient distribuées aux élèves du Séminaire. A. Gosselin, op. cit., 3e partie, Québec, 1914, p. 150 et note 17.

44 Durand de Maillane, Dictionnaire de droit canonique, t. IV, Lyon, 1770 , p. 609 et suiv.

45 Sur les revenus de l'évêque, voir J. BLaIN, L'Église de la NouvelleFrance, 1632-1675, thèse mss, Univ. d'Ottawa, 1967, p. 386 et suiv. Sur tout le problème des finances de l'Église et des subventions destinées à l'entretien du clergé de Nouvelle-France, de bonnes indications dans G. FréGAULT, Le XVIIIe siècle canadien, Montréal, 1968, pp. 134-158. 
Régime français - que nous voyons figurer dans les registres d'insinuations de l'époque ${ }^{46}$.

En cela, la situation des jeunes ordinands séculiers ne différait pas tellement de celle de leurs homologues réguliers, tels les Récollets par exemple, qui étaient ordonnés, conformément aux règles canoniques existantes, sub titulo paupertis. Ni les uns ni les autres n'avaient à donner de garanties financières comparables à celles que l'on exigeait, nous l'avons vu, dans les communautés de femmes. Si l'on ajoute à cela le fait que les Récollets et, jusqu'à un certain point, le diocèse de Québec, n'étaient pas très exigeants quant à la préparation intellectuelle de leurs sujets ${ }^{47}$, on finit par s'expliquer que tant de fils du peuple aient tout de même réussi à accéder au sacerdoce.

Une difficulté reste cependant et à laquelle, dans l'état actuel de nos connaissances, nous ne sommes probablement pas en mesure d'apporter une réponse, du moins une réponse définitive. Elle concerne surtout nos communautés de moniales et nous est suggéré par une remarque de Micheline Dallaire dans son étude sur l'Hôpital Général de Québec à l'effet que les familles ayant souvent le plus de difficulté à rencontrer les obligations de la dot étaient, non pas, comme on pourrait le croire, celles d'origine modeste, mais celles d'élite, et que les suppléments de dots offerts à la communauté allaient beaucoup plus souvent à ces dernières qu'aux autres ${ }^{48}$. D'où la question qui vient immédiatement à l'esprit : l'argent, dans la mesure où il était un obstacle, l'était-il seulement pour les filles du peuple, ou ne l'était-il pas également ou, peut-être même, surtout pour celles de «bonnes familles»?

Notons, tout d'abord, que la plupart des exemples donnés par Micheline Dallaire concernent des familles appartenant à ce qu'elle appelle «l'élite de fonction ou de dignité 49 ». C'est d'ailleurs le groupe qui fournit proportionnellement le plus de recrues $(35,5 \%)$ à la communauté de l'Hôpital Général ${ }^{50}$. Qu'il y ait eu des cas de réelle pauvreté dans ces milieux, son étude semble le démontrer.

46 Renseignements fournis par M. Armand Gagné, archiviste de l'archidiocèse de Québec.

47 N. Baillargeon, Le Séminaire de Québec de 1685 à 1760, pp. $209-$

210. G. Filteau, La naissance d'une nation, t. I, Montréal, 1937, p. 167.

48 M. Dallaire, L'Hôpital-Général de Québec, pp. 116-117.

49 Ibid., p. 55.

50 Ibid., p. 83. 
Mais, comme elle le signale, on tenait à avoir des vocations de cette « qualité » chez les Sœurs de l'Hôpital Général, tout comme à l'Hôtel-Dieu et chez les Ursulines d'ailleurs, et on finissait toujours par trouver l'argent nécessaire, car, nous dit Micheline Dallaire, les bienfaiteurs, dans ces cas, étaient plus faciles à convaincre. "Plaie d'argent n'est point mortelle »: à pauvreté égale, les recrues de "bonnes familles » jouissaient donc, du moins à l'Hôpital Général, d'un net avantage sur celles de modeste ou d'humble extraction. Car, dans pareils cas, ces dernières étaient habituellement reçues comme converses, sans dot fixe, avec des contributions ne dépassant pas en moyenne les 360 livres ${ }^{51}$. Mais on procédait de la même façon chez les Ursulines de Trois-Rivières, chez les Ursulines et les Sœurs de l'Hôtel-Dieu de Québec, si bien que dans ces quatre communautés, les seules de fait à avoir conservé la distinction religieuses de chœur - converses en Nouvelle-France, on comptait $93 \%$ de sœurs de cette dernière catégorie - soit 80 sur 86 provenant de milieux populaires.

ORIGINE SOCIALE DES SEEURS CONVERSES

\begin{tabular}{lccccc}
\hline & $\begin{array}{c}\text { Hôtel-Dieu } \\
\text { de Q. }\end{array}$ & $\begin{array}{c}\text { Hôpital G. } \\
\text { de Q. }\end{array}$ & $\begin{array}{c}\text { Ursulines } \\
\text { de Q. }\end{array}$ & $\begin{array}{c}\text { Ursulines } \\
\text { de T.-R. }\end{array}$ & Ensemble \\
Notables & 5 & 0 & 1 & 0 & 6 \\
Peuple & 25 & 19 & 27 & 9 & 80 \\
$\quad$ Total & 30 & 19 & 28 & 9 & 86 \\
\hline
\end{tabular}

Mais, fait significatif, ce phénomène est à peu près exclusif aux moniales et, on l'aura noté, aux moniales des régions de Québec et de Trois-Rivières. Ces dernières seraient-elles restées plus longtemps fidèles au modèle social importé de France, alors qu'à Montréal, ville-frontière, on s'en serait plus rapidement éloigné ? La chose est possible, du moins en ce qui concerne nos communautés de religieuses, Les deux familles les moins conformistes de ce point de vue, soit les Sœurs de la Congrégation et les Sœurs de la Charité, ne sont-elles pas précisément, toutes deux, des fondations montréalaises ? Encore faudrait-il démontrer qu'à l'intérieur de ces communautés, une fois passé le seuil de l'admission et de la pro-

$51 \quad$ Ibid., p. 106. 
fession, les distinctions sociales ne reprenaient pas leurs droits. De quels milieux venaient les supérieures et les conseillères des deux familles en question? Les filles de notables y étaient-elles mieux traitées et jouissaient-elles de plus de considération que les autres?

Nous savons que chez les Sœurs de la Congrégation, sur 19 conseillères élues et réélues, presque toutes à plusieurs reprises, avant 1763,8 , soit $42 \%$, étaient filles de notables et 11 , soit $58 \%$, filles du peuple. Nous savons également que sur 9 supérieures choisies durant la même période, elles aussi pour plus d'un terme, 4 ou $44 \%$ appartenaient au premier groupe et 5 ou $56 \%$, au second 52 . Ce qui correspond assez bien à la répartition sociale relevée plus haut pour l'ensemble de la communauté, avec un léger avantage toutefois pour les recrues de «bonnes familles». Mais il faudrait étendre cette enquête aux autres communautés de femmes, de même qu'à l'ensemble des établissements ecclésiastiques de l'époque.

Pour l'instant, retenons ce fait, tout de même capital, que l'accès à une vocation religieuse ou sacerdotale en Nouvelle-France semble avoir été, en général, assez peu conditionné par le rang social, si ce n'est dans le cas de quatre communautés, toutes cloîtrées, qui, nous l'avons vu, avaient tendance à réserver la vocation, évidemment moins estimée, de converses aux filles du peuple. Mais, encore là, il faudrait bien se garder d'exagérer, car bon nombre de filles de petits commerçants, artisans ou « habitants » réussirent tout de même, dans ces quatre familles, à se faire admettre comme religieuses de chœur. Bien plus, dans trois d'entre elles, ces religieuses représentent la majorité des effectifs d'extraction populaire : à l'Hôtel-Dieu de Québec, 41 sur 66 ; à l'Hôpital Général, 21 sur 40 ; chez les Ursulines de Trois-Rivières, 12 sur 21. Seules les Ursulines de Québec font exception à la règle, avec 27 converses sur 42 recrues d'humble origine.

Le préjugé social, pour réel qu'il fût dans ces communautés, n'en était pas moins tempéré par d'autres considérations qu'il serait intéressant de connaître un peu plus en détail. Sans doute y subissait-on, consciemment ou inconsciemment, l'influence du modèle social qui était en train de s'élaborer en Nouvelle-France et en ce sens une étude plus poussée du recrutement dans ces communautés, du type de celle qu'a tentée Micheline Dallaire pour l'Hôpital Gé-

52 D'après Sr HeNRIETTE, op. cit., t. I-III, passim. 
néral de Québec ${ }^{53}$, permettrait peut-être de révéler en quoi ce modèle restait fidèle et en quoi il s'opposait à celui de la métropole.

Mais le plus intéressant et le plus révélateur en ce domaine serait peut-être de se pencher sur le cas des 89 filles du peuple qui réussirent à se faire accepter, dans les quatre communautés mentionnées, comme religieuses de chœur. N'aurions-nous pas là un indice, partiel certes, mais non moins valable, de mobilité sociale? En d'autres mots, à travers ces 89 religieuses, ne serait-il pas possible d'atteindre un certain nombre de familles qui commençaient, à l'époque, leur ascension sociale et, par le fait même, arriver à démontrer et à comprendre un peu mieux certains des mécanismes de la mobilité sociale en Nouvelle-France ? Et si, toujours dans la même perspective, nous étendions notre regard à l'ensemble des vocations d'origine populaire, masculines aussi bien que féminines, ne pourrions-nous pas en tirer des indications, plus déterminantes encore, non plus seulement sur le problème de la mobilité, mais sur celui, tout aussi difficile à cerner, de la stratification sociale propre à la colonie ?

Le seul fait, par exemple, que des fils et des filles du peuple y aient eu accès, dans la proportion que nous avons vue, à une vocation sacerdotale ou religieuse, ne devrait-il pas être considéré comme une preuve d'exceptionnelle mobilité sociale ? Et le projet de vie de ces mêmes fils et filles du peuple, comment ne pas croire qu'il n'ait pas été, au moins en partie, influencé par les possibilités de réalisation et de promotion sociales qu'offrait en Nouvelle-France une vocation d'Église.

L'étude récente de Fernand Ouellet sur les officiers de milice à laquelle nous avons fait allusion plus haut donne à penser que ce processus était également et peut-être surtout conditionné par les liens de parentèle et de clientèle existant dans la colonie. L'auteur insiste en particulier sur la force de cohésion et de promotion de la grande famille - de la grande famille rurale surtout, "produit d'une très haute natalité et du foisonnement des mariages dans le milieu immédiat et ses environs ${ }^{54} \gg$. Nous croyons avec lui, à la lumière d'un certain nombre de pointage que nous avons pu faire ${ }^{55}$,

53 Op. cit., p. 51 et suiv.

54 F. OUellet, art. cit., p. 17.

55 Un premier pointage nous a en effet permis d'établir qu'une douzaine de familles (Boucher, Le Gardeur, Gagnon, Leneuf, Juchereau, etc.) monopolisaient, à elles seules, plus de $30 \%$ de toutes les vocations. 
que c'est là un facteur de toute première importance pour nos vocations d'Église aussi bien que pour ses vocations miliciennes. Aussi est-ce dans cette direction surtout que nous entendons poursuivre et, si possible, faire progresser la présente recherche dont nous n'avons pu, de fait, présenter ici qu'un premier et, à vrai dire bien modeste volet. Le dossier reste ouvert et il nous réserve, sans doute, d'autres surprises.

Pierre Hurtubise, o.m.i.,

Université Saint-Paul,

(Ottawa). 